\title{
Agranulocitosis secundaria al uso de vasoprotectores
}

\author{
S. Membrado Gómez, G. Vázquez Perfecto, Mạ M. Álvarez Esteban ${ }^{1}$ \\ Residente $3^{*}$ Año Medicina Familiar y Comunitaria. 'Especialista en Mediana \\ Familiar y Comunitaria. Centro de Salud Palacio Segovia. Área 7. Madrid
}

\section{RESUMEN}

La presencia de agranulocitosis se demuestra por una concentración de neutrófilos en sangre menor de 500 por $\mathrm{mm}^{3}$. Presentamos el caso de un varón de 88 años que desarrolló una agranulocito sis después de tomar dobesilato cálcico y troxeruti na como tratamiento para su retinopatía diabética. Aunque solamente hay descritos casos esporádicos de agranulocitosis por uso de dobesilato y ninguno con la troxerutina, la eficacia de estos vasoprotec tores no ha sido demostrada. Dado que la agranu locitosis tiene una mortalidad de aproximadamente un $15 \%$, consideramos que antes de la prescrip ción de estos fármacos deberían sopesarse sus riesgos y beneficios.

Palabras clave: Dobesilato cálcico. Troxerutina. Agranulocitosis.

\section{Agranulocytosis due to phlebotonics}

\begin{abstract}
The presence of agranulocytosis is signalled by a concentration of neutrophils lower than 500 per $\mathrm{mm}^{3}$. We present the case of an 88 years old man who suffered an agranulocytosis after a treatment with calcium dobesilate and troxerutin prescribed to him after a diagnosis of diabetic retinopathy. Although there are only a few cases of calcium do besilate related agranulocytosis and none related to troxerutin, the efficacy of these phlebotonics has not been demonstrated. Given that agranulocytosis has a mortality rate of approximately $15 \%$, risks and benefits should be balanced before the pres cription of these drugs.
\end{abstract}

Key words: Calcium dobesilate. Troxerutin. Agranulocytosis.

\section{INTRODUCCIÓN}

Según la Organización Mundial de la Salud, se denomina reacción adversa por medicamentos (RAM) o efecto indeseable a cualquier efecto perjudicial o no deseado debido a la administración de un medicamento, a las dosis utilizadas normalmente en seres humanos, con fines profilácticos, diag- nósticos o terapéuticos o con objeto de modificar una función biológica ${ }^{1}$.

Los trastornos hematológicos inducidos por fármacos son reacciones adversas que se diagnostican con muy poca frecuencia en Atención Primaria. Uno de ellos es la agranulocitosis, que se caracteriza por una disminución importante del número de neutrófilos en sangre $\left(<500\right.$ células por $\left.\mathrm{mm}^{3}\right)$ y que predis- 
pone al desarrollo de infecciones graves. Su mortalidad es de alrededor de un $15 \%$ y su incidencia, cuando es secundaria al uso de fármacos, oscila entre 1,7 y 16 casos por millón de habitantes y año según los grupos de edad y las zonas estudiadas ${ }^{2-5}$.

Además del antecedente de exposición a determinados fármacos y del descenso del número de neutrófilos, la presencia de infecciones agudas acompañadas de fiebre elevada, escalofríos, cefalea y dolor faríngeo, el estudio característico de la médula ósea y la resolución del cuadro en menos de un mes a partir de la retirada del agente inductor, son variables que ayudan a orientar el diagnóstico de agranulocitosis secundaria a fármacos. Por el contrario, la presencia de hepatoesplenomegalia o linfadenopatías generalizadas lo descarta.

Los fármacos más frecuentemente relacionados con la agranulocitosis son antitiroideos, fenotiacinas, antiinflamatorios no esteroideos (AINEs), antidepresivos tricíclicos, citotóxicos y algunos antibióticos (derivados penicilínicos, cloxacilina, cloranfenicol, metronidazol, isoniacida, rifampicina, vancomicina, etc.), pero se han implicado más de 150 sustancias $^{6-8}$. Hasta ahora se sabe muy poco acerca de los factores de riesgo que aumentan la predisposición a este tipo de reacciones adversas aunque se han descrito: la edad (ancianos y recién nacidos), el sexo femenino, la existencia de patología asociada y el uso simultáneo de varios fármacos ${ }^{9}$.

\section{OBSERVACIÓN CLÍNICA}

Paciente varón de 88 años con antecedentes de diabetes mellitus tipo 2 diagnosticada hace un año y en tratamiento con dieta, ex-fumador, cardiopatía isquémica con angor hace 10 años, glaucoma en ojo izquierdo e intervenido de catarata en ojo derecho. En tratamiento habitual con ácido acetilsalicílico, nisoldipino y nitroglicerina transdérmica.

Acude a su oftalmólogo de zona, con motivo de una revisión, que le detecta una retinopatía diabética preproliferativa en el ojo izquierdo, con numerosos focos hemorrágicos dispersos por todo el polo posterior y edema macular asociado. Recomienda tratamiento con dobesilato cálcico y troxerutina a dosis de $1.000 \mathrm{mg} /$ día y $600 \mathrm{mg} /$ día respectivamente.

Tres meses después, acude al centro de salud por un cuadro de fiebre elevada de 10 días de evolución y congestión nasal sin otra sintomatología acompañante. La exploración física fue normal exceptuando fiebre de $38,5^{\circ} \mathrm{C}$ y roncus en base derecha. Con el diagnóstico de infección respiratoria el médico de familia pauta tratamiento con $400 \mathrm{mg}$ de cefixima diarios durante 10 días.
A los 5 días de tratamiento persiste la fiebre elevada por lo que el paciente acude a un servicio de urgencias hospitalarias. No refiere focalidad infecciosa, excepto molestias faríngeas. A la exploración destaca temperatura de $39,8^{\circ} \mathrm{C}$, faringe hiperémica sin exudados, auscultación cardiopulmonar normal, no lesiones cutáneas, no visceromegalias ni adenopatías. Allí se realizaron las siguientes pruebas complementarias: una analítica sanguínea donde destaca como dato patológico una cifra total de neutrófilos de 37 por $\mathrm{mm}^{3}$, una glucemia de $341 \mathrm{mg} / \mathrm{dl}$ y una radiografía de tórax sin infiltrados parenquimatosos. Durante el ingreso se realizaron las siguientes pruebas diagnósticas: serologías para citomegalovirus, virus Epstein Barr y toxoplasma, todas ellas negativas; anticuerpos heterófilos negativos, extensión de sangre periférica con leucopenia, neutropenia, monocitosis y células inmaduras. La biopsia de médula ósea presentó disminución de células granulopoyéticas con reducción de las formas maduras e hiperplasia de células precursoras compatible con agranulocitosis. Durante su estancia hospitalaria se retiraron la troxerutina y el dobesilato cálcico, se realizó tratamiento con antibióticos de amplio espectro y factor estimulante de colonias granulocíticas recuperando las cifras normales de neutrófilos en sangre periférica. Recibió el alta con el diagnóstico de agranulocitosis probablemente secundaria a fármacos.

Pasados tres meses, el paciente consulta de nuevo en la urgencia hospitalaria por un cuadro clínico similar al previo. Se realiza una analítica sanguínea que muestra una cifra total de neutrófilos de 44 por $\mathrm{mm}^{3}$ y una radiografía de tórax donde aparece un pequeño infiltrado en la base del pulmón izquierdo. Se comprueba que había vuelto a reintroducir el mes anterior (según refiere el paciente por indicación de su oftalmólogo) los fármacos probablemente implicados en la producción de la primera agranulocitosis. Se procede al ingreso, se retiran definitivamente la troxerutina y el dobesilato cálcico y se le trata por segunda vez con antibióticos intravenosos de amplio espectro y factor estimulante de colonias granulocíticas con buena evolución, consiguiendo la recuperación de las cifras de neutrófilos y la resolución de la neumonía.

Al confirmarse el diagnóstico, desde el centro de salud, se notifica el caso de agranulocitosis secundaria fármacos tras el uso de dobesilato cálcico y troxerutina, a la Consejería de Sanidad (Servicio de Ordenación y Asistencia Farmacéutica).

\section{DISCUSIÓN}

Entre las posibles causas de neutropenia destacan, junto con los fármacos, las infecciones graves. Ante un síndrome febril asociado a neutropenia hay que descartar un proceso infeccioso viral (her- 
pes, varicela, hepatitis B y C, síndrome de EpsteinBarr, VIH), bacteriano (brucelosis, tuberculosis, salmonelosis) o parasitario (leishmaniosis). Otras posibles causas a tener en cuenta serían déficit nutricionales (vitamina $\mathrm{B}_{12}$, ácido fólico, cobre, hierro), infiltración de la médula ósea, anemia aplásica, colagenosis (sobre todo el lupus eritematoso) e incluso algunas endocrinopatías (hipo e hipertiroidismo, enfermedad de Adisson) ${ }^{10}$.

En cuanto al dobesilato cálcico (sulfonato de 2,5-dihidroxibenceno) y a la troxerutina, se les atribuyen las propiedades de disminuir la permeabilidad capilar, la agregación plaquetaria y la viscosidad sanguínea. También aumentan la relajación del endotelio mediante el aumento de la síntesis de óxido nítrico. Por ello, se utilizan como "protectores capilares" en el tratamiento de procesos tan heterogéneos como: insuficiencia venosa crónica, síndrome prevaricoso, varices (esenciales, secundarias y gestacionales), ortostatismo, hemorroides, fragilidad capilar (debida a arteriosclerosis, hipertensión arterial, diabetes, cirrosis y púrpura vascular), uso de anticonceptivos hormonales y anticoagulantes, petequias espontáneas y equímosis, retinopatía arteriosclerótica, diabética y para el tratamiento y la prevención de hemorragias oculares (indicaciones aprobadas para el uso de estos fármacos) ${ }^{11}$.

El dobesilato cálcico se utiliza a dosis de entre 500 a 1.000 mg/día y hasta 2 g/día en oftalmología. La troxerutina se emplea a dosis de $300 \mathrm{mg} / 8 \mathrm{~h}$ y hasta $3 \mathrm{~g} /$ día en la retinopatía diabética severa.

Como efectos secundarios más frecuentes relacionados con el uso del dobesilato cálcico se han descrito molestias gastrointestinales (único efecto secundario registrado en la ficha técnica de este fármaco), reacciones cutáneas y cefaleas (registrado en la ficha técnica de la troxerutina), fiebre medicamentosa y agranulocitosis, este último de forma anecdótica aunque, según un estudio reciente, el dobesilato cálcico podría haber inducido 56 casos de agranulocitosis en España entre los años 1993 y $1998^{11}$. A fecha de hoy, no hay en la literatura casos descritos de agranulocitosis secundaria al uso de troxerutina.

El periodo de tiempo entre el inicio del tratamiento y el primer síntoma (la fiebre en el caso de nuestro paciente) varía entre 7 días y varios años aunque lo más común es que aparezca entre 29 y 90 días después ${ }^{11}$ (el mecanismo de acción de estos fármacos para producir la agranulocitosis no es por un mecanismo de hipersensibilidad sino por un efecto tóxico directo sobre la médula ósea de los pacientes susceptibles).

En pacientes que se expusieron al fármaco de nuevo, como es el caso del paciente que se presenta, los síntomas aparecen entre 15 y 55 días después $^{11-13}$.
Para el establecimiento de una relación de causalidad entre una reacción adversa y un fármaco se utiliza una modificación del algoritmo de Karch y Lasagna ${ }^{14}$ que contempla los siguientes criterios: secuencia temporal entre el inicio del tratamiento y la aparición de la reacción adversa, que ésta haya sido descrita previamente en la literatura, que el efecto indeseable mejore con la retirada del fármaco, que la reacción aparezca nuevamente ante la readministración del fármaco sospechoso y por último, qua no haya una posible explicación alternativa o que sea menos importante que la relación fármaco-reacción adversa. La reacción adversa estará probada o definida cuando se cumplan los cin$\mathrm{co}^{15}$ (éste es el caso del dobesilato cálcico en el caso presentado). En cuanto a la troxerutina, el cuadro presentado por el paciente no es conocido como efecto indeseable del fármaco, por tanto la relación causa efecto sería condicional y difícil de demostrar aunque podría aumentar el riesgo relativo de agranulocitosis al haberse administrado con un fármaco con el que sí está descrita esa reacción adversa $^{16}$.

Por todo ello, el caso clínico que se presenta puede considerarse una agranulocitosis secundaria al uso de dobesilato cálcico sin poder descartarse totalmente la implicación de la troxerutina. Teniendo esto en cuenta, consideramos que debería hacerse un uso más racional de este tipo de fármacos y que su uso generalizado no está justificado, sobre todo si es desfavorable la relación riesgobeneficio. Hay que recordar que su eficacia clínica no está probada ${ }^{11,17-27}$ y que la agranulocitosis está descrita como efecto secundario del dobesilato cálcico $^{7,11-13,28}$. Puesto que las reacciones adversas son el precio de la prescripción de principios activos, el médico de familia deberá sopesar previamente, los beneficios y los riesgos de su uso.

\section{AGRADECIMIENTOS}

A Isabel Miguel, Francisco Muñoz y Alfonso Renart por la supervisión del artículo. A nuestras compañeras Paula Igual y Nuria Ruiz por su ayuda y su ánimo.

\section{CORRESPONDENCIA:}

Silvia Membrado Gómez

Centro de Salud Palacio de Segovia

C/ Segovia, 4

28005 Madrid

Telfs.: $915419005-915417588$ 


\section{Bibliografía}

1. Laporte JR, Capellá D. Mecanismos de producción y diagnóstico clínico de los efectos indeseables producidos por medicamentos. En: Laporte JR, Tognoní G, eds. Principios de epidemiología del medicamento. $2^{\mathrm{a}}$ ed. Barcelona: Masson-Salvat, 1993. p. 95-109.

2. Arneborn P, Palmblad J. Drug-induced neutropenia-a survey for Stockolm 1973-1978. Acta Med Scand 1982; 212: 289-92.

3. Böttiger LE, Furhoff AK, Holmberg L. Fatal reactions to drugs. A 10 year material from the Swedish Adverse Drug Reaction Committee. Acta Med Scand 1979; 205: 451-6.

4. The design of a study of the drug etiology of agranulocytosis and aplastic anemia. A report from the International Agranulocytosis and Aplastic Anemia Study. Eur J Clin Pharmacol 1983; 24: 833-6.

5. Risks of agranulocytosis and aplastic anemia. A first report of their relation to drug use with special reference to analgesics. The International Agranulocytosis and Aplastic Anemia Study. JAMA 1986; 256: 1749-57.

6. Vallés I, Callol JA. Trastornos hematológicos inducidos por fármacos. FMC 1998; 5: 363.

7. Azaceta G, Saenz-Cusi A, Olave T, Palomera L. Agranulocitosis inducida por dobesilato cálcico: a propósito de un nuevo caso. An Med Interna 2000; 17: 337-8.

8. Maldonado Eloy-García J, Sanz Alonso MA, Fontán Casariego G. Enfermedades del sistema leucocitario. En: Farreras Rozman, eds. Medicina Interna. Madrid: Mosby/Doyma, 1995. p. 1672-83.

9. Weber E, Lawson DH, Hoigné R. Risk factors for adverse drug reactions, epidemiological approaches. $1^{\mathrm{a}}$ ed. Boston, 1990.

10. Robledo Martín E, Barajas Gutiérrez MA, Montejo Martínez C. Alteraciones de la série blanca. FMC 1999; 6: 669-78.

11. Ibañez L, Ballarin E, Vidal X, Laporte JR. Agranulocytosis associated with calcium dobesilate clinical course and risk estimation with the case control and the case population approaches. Eur J Clin Pharmacol 2000; 56: 763-7.

12. Cladera Serra A, Blasco Mascaro I, Oliva Berini E, Ramos Díaz F. Agranulocitosis inducida por dobesilato cálcico. Med Clin (Barc) 1995; 28: 558-9.

13. Garcia Benayas E, García Diaz B, Pérez G. Calcium dobesilate induced agranulocytosis. Pharm World Sci 1997; 19: 251-2.

14. Frick PA, Cohen LG, Rovers JP. Algorithms used in adverse drug event reports: a comparative study. Ann Pharmaco-ther 1997; 31: 164-7.
15. Smith JW, Seidl LG, Cluff LE. Studies on the epidemiology of adverse drug reactions. V. Clinical factors influencing susceptibility. Ann Intern Med 1966; 65: 629-40.

16. Kaufman DW, Kelly JP, Jurgelon JM, Anderson T, Issaragrisil S, Wiholm BE, et al. Drugs in the aetiology of agranulocytosis and aplastic anaemia. Eur J Haematol 1996; 578 (Supl. 60): 23S-30S

17. Widmer L, Biland L, Barras JP. Doxium 500 in chronic venous insufficiency: a double blind placebo-controlled multicentre study. Int Angiol 1990; 9: 105-10.

18. Pecchi S, De Franco V, Damiani P, Guerrini M, Di Perri T. Calcium dobesilate in the treatment of primary venous insufficiency of the lower limbs. A controlled clinical study. Clin Ter 1990; 132: 409-17.

19. Androulakis G, Panoysis PA. Plethysmographic confirmation of the beneficial effect of calcium dobesilate in primary varicose veins. Angiology 1989; 40: 1-4.

20. Casley-Smith JR. A double-blind trial of calcium dobesilate in chronic venous insufficiency. Angiology 1988; 39: 853-7.

21. Vojnikovic B. Doxium (calcium dobesilate) reduces blood hyperviscosity and lowers elevated intraocular pressure in patients with diabetic retinopathy and glaucoma. Ophthalmic Res 1991; 23: 12-20.

22. Leite EB, Mota MC, Faria de Abreu JR, Cunha-Vaz JG. Effect of calcium dobesilate on the blood-retinal barrier. Int Ophthalmol 1990; 14: 81-8.

23. Guerrini M, Pieragalli D, Acciavatti A, Galigani C, Guideri F, Franchi M, et al. Calcium dobesilate, hemorheology, fibrinolysis and endotelium. New perspectives on the prevention of diabetic microangiopathy. Clinical pharmacological study. Clin Ter 1989; 129: 271-85.

24. Vinazer $\mathrm{H}$, Hachen $\mathrm{HJ}$. Influence of calcium dobesilate (Doxium) on blood viscosity and coagulation parameters in diabetic retinopathy. Vasa 1987; 16: 190-2.

25. Paroven : not much effect in trials. Drug Ther Bull 1992; 30 : 7-8.

26. Schuller-Petrovic S, Wolzt M, Böhler K. Studies on the effect of short-term oral dihydroergotamine and troxerutin in patients with varicose veins. Clin Pharmacol Ther 1994; 56: 452-9.

27. Colgan MP, Moore DJ, Shanik DG. New approaches in the medical management of venous ulceration. Angiology 1993; 44: 138-42.

28. Kulessa W, Becker EW, Berg PA. Recurrent agranulocytosis after taking calcium dobesilate. Dtsch Med Wochenschr 1992; 117: 372-4. 